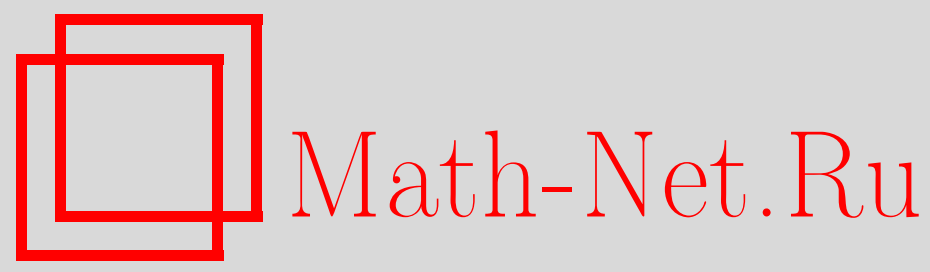

В. А. Юдин, Экстремальные свойства функций и дизайны на торе, Матем. заметки, 1997, том 61, выпуск 4, 637-640

DOI: https://doi.org/10.4213/mzm1544

Использование Общероссийского математического портала Math-Net.Ru подразумевает, что вы прочитали и согласны с пользовательским соглашением http://www.mathnet.ru/rus/agreement

Параметры загрузки:

IP: 54.81 .137 .203

26 апреля 2023 г., 14:57:43

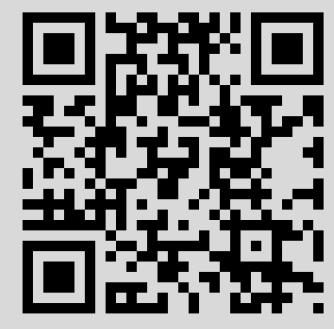




\section{ЭКСТРЕМАЛЬНЫЕ СВОЙСТВА ФУНКЦИЙ И ДИЗАЙНЫ НА ТОРЕ}

\section{В. А. Юдин}

Пусть $x=\left(x_{1}, \ldots, x_{n}\right) \in \mathbb{R}^{n}, x y=x_{1} y_{1}+\cdots+x_{n} y_{n}-$ скалярное произведение $x$ и $y$, $|x|=\sqrt{x x} ; \omega_{n}=\pi^{n / 2} \Gamma^{-1}(n / 2+1)$ - объем единичного шара $B_{n}, B_{n}=\left\{x \in \mathbb{R}^{n}:|x|<1\right\}$; $\mathbb{T}^{n}=\left\{x \in \mathbb{R}^{n}:-1 / 2<x_{k} \leqslant 1 / 2\right\}-n$-мерньй тор; $q_{n}$ - первый нуль функции Бесселя $J_{\nu}(z), \nu=n / 2$. Через

$$
\sum_{\nu \in \mathbb{Z}^{n}} \widehat{f}_{\nu} e^{2 \pi i \nu x}, \quad \widehat{f}_{\nu}=\int_{\mathbb{T}^{n}} f(x) e^{-2 \pi i \nu x} d x
$$

обозначим ряд Фурье функции $f(x)$ из $L\left(\mathbb{T}^{n}\right)$, а через $\operatorname{sp} f=\left\{\nu \in \mathbb{Z}^{n}: \widehat{f}_{\nu} \neq 0\right\}-$ спектр функции $f(x)$. Пусть $D$-центрально-симметрическая область из $\mathbb{R}^{n}$. Множество точек $\left\{x^{(k)}\right\}_{k=1}^{N}=W, W \subset \mathbb{T}^{n}$, назьвается дизайном типа $D$, если кубатурная формула

$$
\int_{\mathbb{T}^{n}} f(x) d x=\frac{1}{N} \sum_{k=1}^{N} f\left(x^{(k)}\right)
$$

точна для любого тригонометрического полинома $f(x), \operatorname{sp} f \subset D$. Это определение эквивалентно выполнению следующих тождеств:

$$
\sum_{k=1}^{N} e^{2 \pi i \nu x^{(k)}} \equiv 0, \quad \nu \in D \cap \mathbb{Z}^{n} \backslash\{0\}
$$

Основная задача состоит в построении дизайнов с наименьшим количеством точек $N$. Первоначальные исследования [1], [2] относятся к случаю, когда область $D$ имеет вид

$$
D=\left\{\nu \in \mathbb{Z}^{n}:\left|\nu_{1}\right|+\cdots+\left|\nu_{n}\right|<s\right\}, \quad s \in \mathbb{N} .
$$

В [3] рассматривается общий случай, получена оценка

$$
N \geqslant \sum_{\nu \in \mathbb{Z}^{n}, \nu \in \frac{1}{2} D} 1, \quad \lambda D=\left\{x \in \mathbb{R}^{n}: \frac{x}{\lambda} \in D\right\}, \quad \lambda>0 .
$$

Близкую оценку

$$
N \geqslant \operatorname{mes} \frac{1}{2} D=\frac{\operatorname{mes} D}{2^{n}},
$$

где $D$ - выпуклая центрально-симметрическая область из $\mathbb{R}^{n}$, можно извлечь из доказательства К. Зигеля теоремы Г. Минковского о целой точке в вьпуклом теле. Аналогичная задача возникает и для других компактов. Наиболее интенсивные исследования

Работа выполнена при финансовой поддержке Российского фонда фундаментальных исследований, грант № 96-01-00212. 
относятся к случаю многомерной сферы (см. известные обзоры [4], [5] с подробной историей вопроса). Последние достижения в оценках снизу мощности дизайнов получены с помощью аналитических методов, основанных на экстремальных свойствах полиномов и функций.

Совокупность непрерьвных на $\mathbb{T}^{n}$ функций, удовлетворяющих условиям

1) $f(x) \geqslant 0, x \in \mathbb{T}^{n}$

2) $\widehat{f}_{0}>0, \widehat{f}_{\nu} \leqslant 0, \nu \in \mathbb{Z}^{n} \backslash D$,

обозначим через $M(D)$.

Рассмотрим сумму

$$
I=\sum_{k, l=1}^{N} f\left(x^{(k)}-x^{(l)}\right)
$$

Из первого условия находим

$$
I \geqslant \sum_{k=l} f\left(x^{(k)}-x^{(l)}\right)=N f(0)
$$

За исключением конечного числа все коэффициенты Фурье функции $f(x)$ имеют одинаковьй знак. Следовательно, ряд Фурье функции $f(x), f(x) \in M(D)$, является абсолютно сходящимся. Поэтому из условия 2) получим

$$
\begin{aligned}
I & =\sum_{\nu \in \mathbb{Z}^{n}} \widehat{f}_{\nu} \sum_{k, l=1}^{N} e^{2 \pi i \nu\left(x^{(k)}-x^{(l)}\right)}=\sum_{\nu \in \mathbb{Z}^{n}} \widehat{f}_{\nu}\left|\sum_{k=1}^{N} e^{2 \pi i \nu x^{(k)}}\right|^{2} \\
& =N^{2} \widehat{f}_{0}+\sum_{\substack{\nu \in \mathbb{Z}^{n} \\
\nu \in D \backslash\{0\}}} \widehat{f}_{\nu}\left|\sum_{k=1}^{N} e^{2 \pi \nu x^{(k)}}\right|^{2}+\sum_{\substack{\nu \in \mathbb{Z}^{n} \\
\nu \notin D}} \widehat{f}_{\nu}\left|\sum_{k=1}^{N} e^{2 \pi \nu x^{(k)}}\right|^{2} \leqslant N^{2} \widehat{f}_{0},
\end{aligned}
$$

так как $\left\{x^{(k)}\right\}_{k=1}^{N}-$ дизайн типа $D$. Из $(2),(3)$ найдем

$$
N \geqslant \frac{f(0)}{\widehat{f}_{0}}
$$

Таким образом, для оценки снизу мощности дизайнов приходим к экстремальной задаче: среди всех функций из класса $M(D)$ найти функцию, доставляющую наибольшее значение отношения (4).

В одномерном случае $D=\{\nu \in \mathbb{Z}:|\nu|<q\}$ хорошо известно, что минимальным дизайном являются $q$ узлов формулы прямоугольников: $x^{(k)}=k / q, k=0,1, \ldots, q-1$. Так что для всякой функции $f(x) \in M(D)$ выполняется неравенство $f(0) \leqslant q \widehat{f}_{0}$, оно не может быть улучшено. Знак равенства достигается для функции $f(x), f(x) \in M(D)$, построенной следуюшим образом:

$$
f(x)=\sum_{k \in \mathbb{Z}} F(x-k), \quad F(x)=\left(F_{1} * F_{2}\right)(x)=\int_{\mathbb{R}} F_{1}(x-y) F_{2}(y) d u, \quad x \in \mathbb{R}
$$


где

$$
F_{1}(x)=\left\{\begin{array}{ll}
\cos ^{2} \pi q x, & |x| \leqslant \frac{1}{2 q}, \\
0, & |x|>\frac{1}{2 q},
\end{array} \quad F_{2}(x)=\left\{\begin{array}{l}
1,|x| \leqslant \frac{1}{2 q}, \\
0,|x|>\frac{1}{2 q} .
\end{array}\right.\right.
$$

В многомерном случае точное решение неизвестно. Для случая $D=R B, R>1$, построим функцию $f(x), f(x) \in M(R B)$, с помощью которой из неравенства (4) найдем новую оценку снизу мощности дизайна. С ростом размерности, $n \rightarrow \infty$, она улучшает известную (1) на экспоненциальньй множитель. Имеет место

ТЕОремА. Для любых $n \in \mathbb{N}, R>1$ выполняется неравенство

$$
N \geqslant(2 \pi R)^{n} \omega_{n}^{-1} q_{n}^{-n}
$$

ДокАЗАТЕЛЬСтво. Для построения функции $f(x)$ из $M(R B)$ будем использовать известную конструкцию из [6], где построена радиальная, непрерьвная функция $W(x)$, $x \in \mathbb{R}^{n}$, с конечным носителем, удовлетворяющая условиям

а) на функцию $W(x)$ :

$$
W(x) \geqslant 0, \quad x \in \mathbb{R}^{n}, \quad W(0)=m \omega_{n} q_{n}^{n}, \quad m=-q_{n}^{n} J_{n / 2}\left(q_{n}\right)
$$

б) на преобразование Фурье [7]:

$$
\widehat{W}(s)=\int_{\mathbb{R}^{n}} W(x) e^{-2 \pi i s x} d x, \widehat{W}(s) \leqslant 0, \quad|s| \geqslant R, \quad \widehat{W}(0)=m(2 \pi R)^{-n} \omega_{n}^{2} q_{n}^{2 n} .
$$

Рассмотрим периодизацию $W(x)$

$$
f(x)=\sum_{k \in \mathbb{Z}^{n}} W(x-k)
$$

Из (6), (8) находим, что $f(x) \geqslant 0, x \in \mathbb{T}^{n}$, и

$$
f(0)=\sum_{k \in \mathbb{Z}^{n}} W(k) \geqslant W(0)=m \omega_{n} q_{n}^{n}
$$

Так как

$$
\begin{aligned}
\widehat{f}_{\nu} & =\int_{\mathbb{T}^{n}} f(x) e^{-2 \pi i \nu x} d x=\int_{\mathbb{T}^{n}} \sum_{k \in \mathbb{Z}^{n}} W(x-k) e^{-2 \pi i \nu x} d x \\
& =\sum_{k \in \mathbb{Z}^{n}} \int_{\mathbb{T}^{n}} W(x-k) e^{-2 \pi i \nu x} d x=\int_{\mathbb{R}^{n}} W(x) e^{-2 \pi i \nu x} d x=\widehat{W}(\nu),
\end{aligned}
$$

то из (7) получаем

$$
\widehat{f}_{\nu} \leqslant 0, \quad|\nu| \geqslant R, \quad \widehat{f_{0}}=\widehat{W}(0)=m(2 \pi R)^{-n} \omega_{n}^{2} q_{n}^{2 n} .
$$


Таким образом, построенная функция $f(x)$ принадлежит классу $M(R B)$. Оценим снизу отношение $f(0) / \widehat{f}_{0}$. Из $(9),(10)$ находим

$$
\frac{f(0)}{\widehat{f}_{0}} \geqslant \frac{m \omega_{n} q_{n}^{n}}{m(2 \pi R)^{-n} \omega_{n}^{2} q_{n}^{2 n}}=(2 \pi R)^{n} \omega_{n}^{-1} q_{n}^{-n} .
$$

Используя неравенство (4) получаем (5). Теорема доказана.

Для первого нуля $q_{n}$ функции Бесселя $J_{n / 2}(z)$ известно [8] асимптотическое поведение

$$
q_{n}=\frac{n}{2}(1+o(1)), \quad n \rightarrow \infty
$$

Дополнительно привлекая асимптотику гамма функции при больших значениях аргумента, находим, что

$$
N \gg\left(\frac{8 \pi}{e n}\right)^{n / 2} R^{n}
$$

Скорость роста написана в экспоненциальной шкале. Степенные множители не учитываются. Неравенство (11) улучшает известную (1) с $D=R B$ оценку в $(4 / e)^{n}$ раз.

При фиксированной размерности $n$ и $R \rightarrow \infty$ существование дизайнов порядка $R$ с количеством точек $R^{n}$ (по порядку) очевидно. Однако, при $n \rightarrow \infty$ точная зависимость от размерности неизвестна.

Московский энергетический институт

Поступило

15.04 .96

\section{СПИСОК ЦИТИРОВАННОЙ ЛИТЕРАТУРЫ}

1. Мысовских И. П. // Докл. АН СССР. 1987. Т. 296. №1. С. 28-31. 2. Beckers M., Cools M. // Inter. Series of Numer. Math. 1993. V. 112. P. 13-24. 3. Резцов А. В. // Матем. заметки. 1990. Т. 48. №1. С. 151-152. 4. Reznick B. // Mem. Amer. Math. Soc. 1992. V. 96. № 463. 5. Seidel J. // Diskrete Math. 1994. V. 136. P. 281-293. 6. Юдин В. А. // Дискретная матем. 1989. Т. 1. №2. С. 155-158. 7. Стейн И., Вейс Г. Введение в гармонический анализ на евклидовых пространствах. М.: Мир, 1974. 8. Ватсон Г. Теория бесселевьх функций. М.: ИЛ, 1949. 